\title{
On Expert Performance in 3D Curve-Drawing Tasks
}

\author{
Ryan Schmidt ${ }^{1,2}$, Azam Khan ${ }^{2}$, Gord Kurtenbach ${ }^{2}$, and Karan Singh ${ }^{1}$ \\ ${ }^{1}$ Dynamic Graphics Project, University of Toronto, Canada \\ ${ }^{2}$ Autodesk Research, Toronto, Canada
}

\begin{abstract}
A study is described which examines the drawing accuracy of experts when drawing foreshortened projections of $3 D$ curves in ecologically-valid conditions. The main result of this study is that the distribution of error in expert drawings exhibits a bias similar to that previously observed in non-expert subjects, which is dependent on the degree of foreshortening of the imagined drawing surface. A review of existing perceptual studies also finds that only absolute 2D image-space error has been considered, which has been found to be largest with viewing angles of $25-55^{\circ}$. Our visualizations of $3 D$ error indicate that $3 D$ bias continues to increase with decreasing viewing angle. Based on these findings, we analyze current $3 D$ curve drawing techniques for susceptibility to foreshortening bias, and make suggestions for future sketch-based modeling systems.
\end{abstract}

Categories and Subject Descriptors (according to ACM CCS): I.3.3 [Computer Graphics]: Picture/Image Generation-Line and curve generation

\section{Introduction}

Inference of 3D lines and curves from sketched 2D strokes is perhaps the most fundamental problem in sketch-based 3D interfaces. The simplest approach to "inverting the projection" of a sketch is to asssume that it represents planar silhouette curves lying parallel to the image plane [IMT99, SWSJ05, KH06]. Virtually every other technique that has been proposed, from drawing directly onto arbitrarilyoriented 3D planes [GBK ${ }^{*}$ 02,TBSR04,Kal05,DXS*07] and surfaces [IMT99, OSD06, NISA07], to sketching connected linear and curved edges of 3D components [Pug92, ZHH96, VTMS04, ML05, LFG08, CKX*08, Goo09], to direct 3D interpretation of sketched freeform curves [CMZ*99, KHR04, KS07, BBS08], assumes that the sketched strokes are foreshortened projections of 3D curves.

Compared to drawing in the image plane, drawing foreshortened projections of $3 \mathrm{D}$ curves is qualitatively more difficult. Consider drawing a wireframe cube in which one face is aligned with the image plane. While the front-facing square can be precisely constructed with a ruler and compass, no such tool exists to find the correct length of the receding edges, which can only be visually estimated.

It is generally accepted that any sketch will have mechanical error or noise, resulting in noisy 3D inference. But er- rors made when estimating the projected shape of foreshortened curves are qualitatively different. In the case of the wireframe cube, the length of the receding lines determine whether the result is a cube, as the artist intended, or a rectangular box. We would refer to the latter as an error of intent. In our experience, error of intent is much more frustrating than mechanical error. We can clearly see that our strokes are noisy and do not correspond to what we meant to draw, so noisy 3D results are to be expected. But errors of intent cannot be predicted from our 2D strokes - they are only discovered once the camera is rotated, and we find that our carefully-drawn cubes are not cubes at all (Figure 1).

It is often argued that SBM systems are meant for "conceptual" stages of design, where ideation and communication is more important than precision, and so the noise introduced by drawing errors will be tolerated. However, errors of intent can be much more severe than mechanical error. A designer may allow for tires of a car that are not perfectly circular, but if they are clearly ellipses, then the wrong intent is communicated.

If errors of intent were normally distributed, we could expect that they would "average-out" over the drawing, particularly if the artist over-sketched each stroke a few times. However, existing literature in areas of visual perception, psychology, and neuroscience provide extensive evidence 


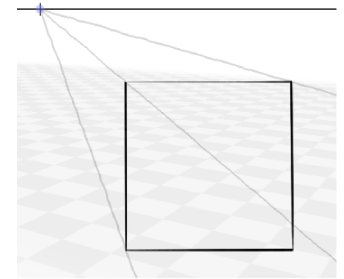

(a)

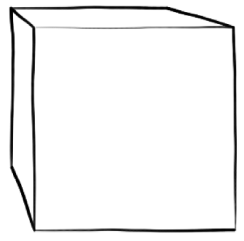

(b)

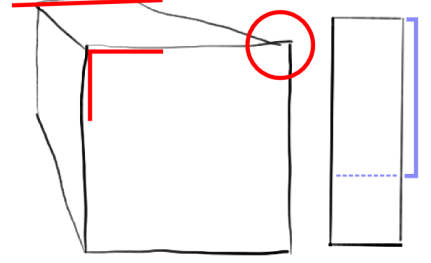

(c)

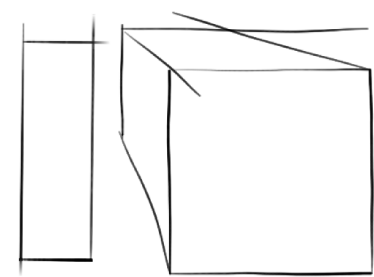

(d)

Figure 1: In this pilot test, subjects were provided with a square and 3 vanishing lines, and asked to draw the receding edges of a cube (a). The correct solution is shown in (b). Results from two subjects $(c, d)$ are shown in frontal and top-down views. The magnitude of mechanical error, such as deviations from straight lines, precise angles, and well-defined junctions (highlighted in red), varies between subjects depending on drawing skill. The error of intent (blue highlight) made in estimating the correct length of the receding lines is very large, and also remarkably consistent between subjects.

that the distribution of error in our our perception of information has a fundamental bias which is somehow related to our prior experience with the visual world. In particular, works which have studied the perception of 3D line drawings, and the errors humans make when depicting 3D objects with line drawings, have found a remarkably consistent bias when the lines and curves involved are foreshortened due to parallel or perspective projection. Furthermore, this bias appears to be dependent not only on the projection in use, but also on the particular shape of the underlying 3D objects.

The perception literature suggests that these biases are fundamental and not affected by the skill of the subject. In reviewing existing studies, we have found that while "regular" untrained subjects are well-represented, and subjects with general artistic training are sometimes included, no existing studies consider 3D drawing "experts". As shown in Figure 2, expert 3D artists appear to be drastically more accurate than even those with general artistic training. Although foreshortening bias is undoubtedly still present, we questioned whether the magnitude of these errors might be diminished to the level of mechanical noise.

Furthermore, existing studies focus on a few very simple depiction tasks, often in highly controlled conditions (to the point where subjects heads are fixed in place). These findings may not be representative of the magnitudes of error a SBM system would encounter in more realistic situations. To explore these issues, we performed a drawing study with trained 3D depiction experts, using unconstrained penciland-paper drawing conditions to avoid any adverse influence on the drawing process. Our results show that even experts exhibit biased depiction errors of significant magnitude.

Despite being highly relevant to sketch-based 3D interfaces, and to computer graphics in general, before undertaking this work the authors were not aware of the fundamental bias in perception and depiction of foreshortened projections of 3D geometry. Furthermore, although painfully aware of our own poor drawing skills, the authors did assume that expert artists were capable of accurately "draw- ing what they meant". Hence, the goal of this paper is to introduce these issues to the SBM research community, and demonstrate that even curves sketched by highly trained design drawing experts will contain errors of large magnitude and consistent bias. Knowledge of this bias can help us to differentiate between which depiction errors are due to the artist having insufficient training, and which are fundamental and must somehow be compensated for even in expert interfaces. Hence, based on our findings we draw some implications for the design of future SBM tools.

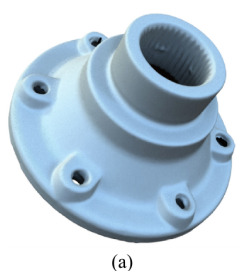

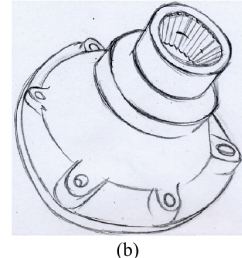

(b)

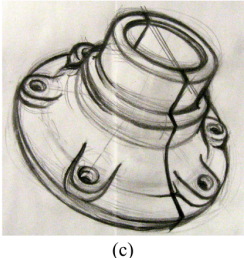

(c)
Figure 2: One of the stimulii (a) and the (subjectively) most accurate freehand sketch $(b)$ created by a participant in a drawing study [CGL ${ }^{*}$ 08] (Images (c)Forrester Cole [CGL*09]). Note the gross errors in shape and proportion. A freehand drawing by an artist with design-drawing training (c) is much more accurate (Image (C)Seok-Hyung Bae).

\section{Perception and Depiction of Projected 3D Curves}

There is a vast amount of literature in visual perception, psychology, and neuroscience that can inform the design of SBM interfaces, shape modeling tools, and computer graphics research in general. In this section we briefly survey a subset of this work that focuses on the perception and depiction of line drawings of projected 3D geometry. These studies indicate that humans make systematic errors both when depicting and perceiving the shape of $3 \mathrm{D}$ objects from foreshortened viewpoints.

Taylor and Mitchell [TM97] provide an illustrative exam- 
ple. A glow-in-the-dark circular disc was presented to subjects at several angles, producing elliptic silhouettes. The stimulus was viewed through a pinhole in an otherwise black box, eliminating perceptual cues. In the control condition, subjects were presented with several ellipses and asked to match the shape using an interactive computer tool. Next the same test was performed, but subjects were first shown that the true shape of the stimulus was a circular disc. In this case, despite identical stimulus, subjects consistently exaggerated the circularity of their ellipses. Artists tested separately produced similar error, indicating that this is not an issue of skill, but a fundamental human limitation (See Figure 3 for a somewhat mind-boggling example). This perceptual interference caused by knowledge of true 3D shape has been observed repeatedly [RL95, MRAR05, MA08], and errors of up to $20 \%$ have been reported [TM97,HKJR08]. A recent study found that the largest errors occur in the viewingangle range of $25-55^{\circ}$ [HKJR08], often considered 'representative' views by artists [GRMS01].
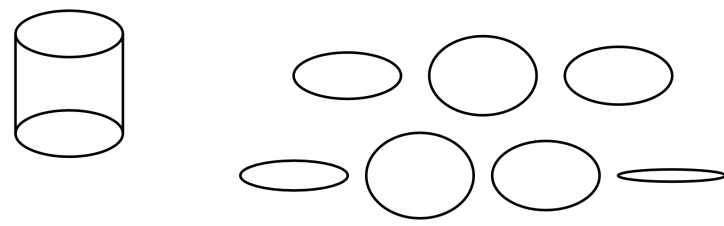

Figure 3: When asked to select the ellipse which matches the top of the cylinder, most humans will choose the top-right ellipse, whereas the correct answer is the top-left ellipse.

In attempting to determine the source of drawing errors, Cohen and Bennet [CB97] ruled out representational choices, motor coordination limitations, and accuracy selfassessment as factors, which left only misperception of the object. Kozbelt [Koz01] found that while artists are much more accurate than non-artists at reproducing abstract $2 \mathrm{D}$ line drawings, independent judges found no significant difference in accuracy between the subject pools when drawing a pair of scissors.

Two large studies by Nicholls \& Kennedy [NK93, NK95] found that when drawing the receding edges of a front-facing cube, subjects strongly preferred specific edge directions and lengths. We repeated this experiment using a 3D drawing tool (Figure 1). Even when the correct vanishing lines are provided, pilot subjects made highly similar errors in their judgement of the foreshortened depth dimension.

From these and other findings, it has been theorized that our internal canonical representation of the true 3D cylinder, which has a circular cap, somehow contaminates our ability to correctly perceive or depict the shape. This is blatantly obvious in children's drawings, and while the effect consistently decreases at several developmental stages [Too99], it does not seem to ever completely disappear. A recent study even found that in a cylinder drawing task, canonical representations vary between subjects and correlate with errors made in drawing a real cylinder [MA08].

Recent work in statistical neuroscience has attempted to explain these perceptual phenomena in terms of probability distributions in the natural world. For example, [NLC*00] samples the subtense of all possible 3D source angles under perspective projection, showing that bias in the resulting distributions corresponds to angular error in estimations of subjects. More recent work has collected distributions based on range scans of real-world scenes, showing that biases in perception of intersecting contours [HP05] and depth [YP03] can be predicted from natural statistics.

The general conclusion implied by these studies is that our prior experience of the physical world causes us to make biased assumptions when perceiving visual stimulus. Particularly relevant to sketch-based design systems is that knowledge of true 3D shape causes humans to make persistent errors when drawing foreshortened projections of 3D curves.

\section{Experiment}

The results described in the previous section have clear implications for the task of inferring 3D geometry from sketches. If the projections of the intended $3 \mathrm{D}$ curves cannot be drawn correctly, then upon rotating the view, the designer will discover that the $3 \mathrm{D}$ curve is not the intended shape. Hence, errors of intent are inherent and unavoidable, and furthermore can be of significant magnitude. But when considering this argument, and the body of work which supports it, we are left with a few questions.

First, the studies that have quantified or visualized error focus on specific cube and cylinder drawing conditions, and only measure error as deviation in the image plane. It is unclear how this translates into 3D error, particularly for more complex 3D curves. Furthermore, rigorous perceptual studies attempt to minimize all contextual cues, but SBM systems usually involve drawing in the context of existing geometry and visual aids. This context may help to mitigate perceptual biases. Hence, it is important to explore the magnitude of error in 3D shape resulting from common inference techniques in real-world drawing conditions.

Second, while some studies do include subjects with general art-school training, Kozbelt [Koz01] notes that such subjects (who are usually students) focus mainly on abstractions or purely $2 \mathrm{D}$ subject matter. Accurate $3 \mathrm{D}$ depiction is largely the domain of architects and industrial designers, who have not been specifically considered by perceptual studies. As shown in Figure 2, this population appears to be much more accurate than the average artist when drawing projections of 3D objects. It does seem plausible that designers may have honed their perceptual skills, "training away" perceptual bias and allowing them to draw projections more accurately. After finding in informal experiments that trained designers 
were more accurate in the ellipse test (Figure 3), we felt that further exploration of this possibility was warranted.

\subsection{Experimental Set-Up and Procedures}

We performed an experiment to analyze the magnitude of 3D errors that are observed when expert subjects sketch projections of 3D curves from foreshortened viewpoints. We recruited 12 subjects who are involved in the creation of 3D design software. All subjects had post-secondary training in art or design, including 6 in architecture and industrial design, 5 of which had some level of professional experience. Several subjects did note that they do not draw regularly, but as product designers and testers, they all interact with computer projections of $3 \mathrm{D}$ curves on a day-to-day basis.

While our original intent was to conduct the study using a pen-input display, in pilot tests we found that our software interface had a detrimental effect on the drawing style of subjects. It seems that the physical properties of the pen-input device, combined with the limited feedback rate and antialiased-line rendering style of our drawing tool, prevented testers from drawing naturally. To avoid any adverse effects from these constraints, we instead adopted a paperbased protocol which provided affordances more comfortable to experienced artists.

Subjects were provided with a booklet of 26 images, each $7.25 \mathrm{in}^{2}$ on a $8.5 \times 11$ in page, rendered from 3D scenes using Maya. Subjects were instructed to draw the projection of a specific 3D curve on each page, as accurately as possible. Drawing was unsupervised, and no time limit was enforced, so subjects were free to draw at their own pace. No restrictions were placed on drawing, subjects were permitted to oversketch or erase and redraw the curve until they were satisfied. We did ask that they trace over their "final" curve with a darkened line, but this instruction was often ignored.

Subjects did oversketch extensively, and in some cases drew many additional guidelines. This noise made it unlikely that any automated curve-fitting technique would provide coherent results, so the data was manually processed. We carefully aligned each page with alignment guides in the 3D scene, over top of a pen-input display. The subject's curve was then projected back onto the 3D surface by carefully tracing it in Maya. [CB97] ruled out perceptual biases in tracing tasks, and to account for mechanical tracing error, each curve was traced a minimum of 3 times, with any significant errors discarded. In cases where the subject had oversketched extensively but not indicated a specific curve, multiple representative curves were traced. In random sampling, an independent judge agreed that the traced curves were of high accuracy.

Although each image was presented only once, viewpoints were re-used and we did not vary presentation order, so some learning effects may be present. Since we provided no feedback on drawing accuracy, we expected that these effects would be small relative to the magnitude of errors we observed in pilot experiments. We also could not control viewing conditions, so subjects are unlikely to have viewed the drawings from the correct center-of-projection. Although this does introduce additional perceptual bias [Tod08], it is an inherent factor in virtually all projective viewing.
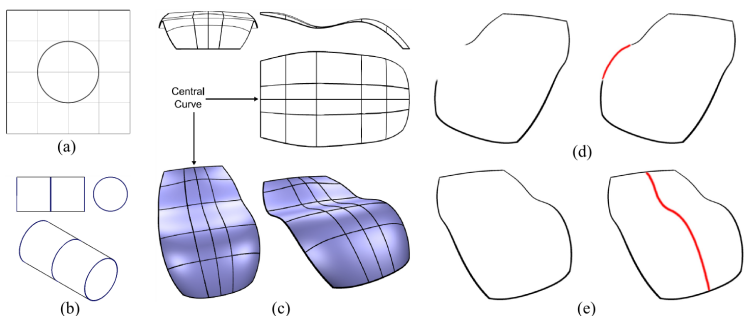

Figure 4: In Part 1 of our study, subjects drew a circle $2 \times 2$ units centered in a $4 \times 4$-unit square (a), and a circle halfway along a 1x1x2 unit cylinder (b) from four foreshortened perspective viewpoints. Next they were given a sheet depicting a mirror-symmetric $3 D$ surface (c) and asked to fill in a missing portion of the symmetric outline in Part $2(d)$, then draw the central curve lying on the surface in Part $3(e)$.

\subsection{Results and Discussion}

We face a problem encountered by previous drawing studies [CB97, Koz01], namely that there is no clear definition of "accuracy" which can be used to objectively quantify our results. However, even simple visual inspection of the data allows meaningful conclusions to be drawn. In the following figures, subject strokes are shown in red, and the target curve in black. We emphasize that high-frequency wiggles in the curves are largely due to the discrete sampling rate of the pen-input digitizer, which is exaggerated with increased foreshortening. We are interested only in the general shape and location of the curves, which is representative of the subject drawings. To make general trends more visible, we apply a slight blur to the aggregate subject strokes.

In the first part of our study, subjects drew a circle on a foreshortened 3D square and cylinder (Figure 4a-b). Small orthographic views were provided indicating that the circle was meant to be centered on the square and half its width, while the cylinder was one unit wide by two units long and the circle should be drawn half-way along the receding axis. Each case was presented from four viewpoints on separate pages, shown with the projected data in Figure 5.

For the circle-on-plane conditions, roughly half the subjects used geometric guideline techniques to construct the circle with reasonable accuracy (in some cases it appears that rulers were used). While this did subvert our experimental goals, it also increased our confidence that subjects were in fact trying to draw as accurately as possible. Regardless, there is a visible bias towards the near side of the 
plane which increases as the foreshortening becomes more extreme, as does the deviation from circularity. In the cylinder condition this effect is even more apparent, it is clear that subjects' intuition for the point at which to split the cylinder edge is biased towards the viewpoint. The "C"-shape of the projected curves indicates that subjects have difficulty determining how circular to draw the internal arc - the 2D strokes are "flatter" than the correct result.

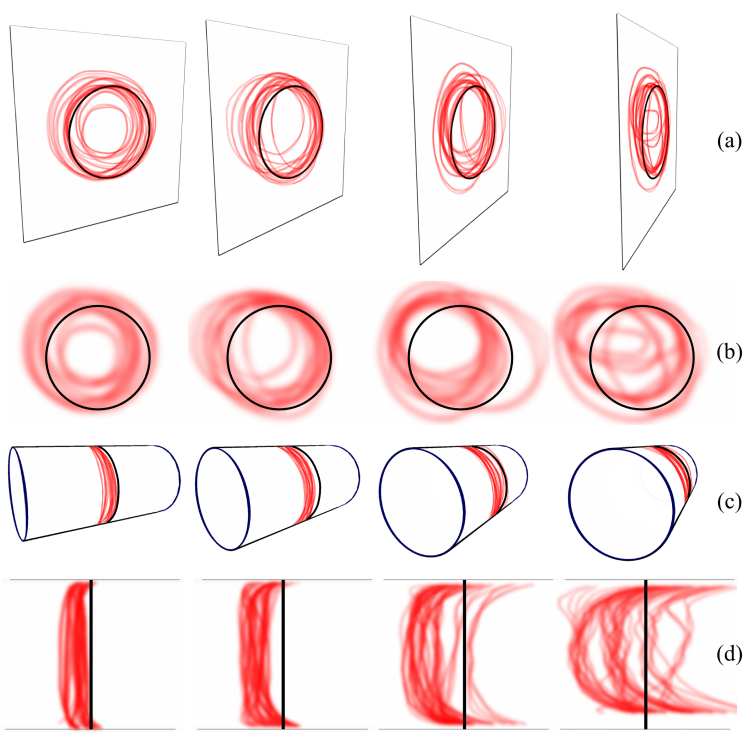

Figure 5: The four plane viewpoints are shown in (a), with the projected curves in red and the correct circle in black. A facing-view (b) shows a clear bias towards the front of the plane, and slightly towards the top. Note that in (b) curves which were analytically constructed with guidelines have been discarded. In the cylinder case $(c, d)$ we see an even clearer bias towards the viewpoint.

Next subjects were given a sheet of paper depicting a freeform 3D surface which was mirror-symmetric about a central plane (Figure 4c). We then presented 3 outline views of this shape with a portion of the outline missing, and asked the subjects to complete the curve, reminding them of the mirror-symmetry (Figure 6a-c). This case was inspired by the ILoveSketch system [BBS08], which provides a tool for inverting the perspective projection of a symmetric pair of sketched curves based on epipolar constraints. Since this involves a parameterized energy minimization over smooth splines fit to each curve, a wide range of 3D results are possible, and hence we present only image-based drawing error. As subjects could reasonably approximate the target curve by smoothly interpolating between the tangent vectors at the provided boundary conditions, we expected quite accurate performance in this condition. However, there is a clear bias in the curves towards the line connecting the contour endpoints, particularly in the third view. Subjects again drew 2D curves which are "flatter" than the correct result.
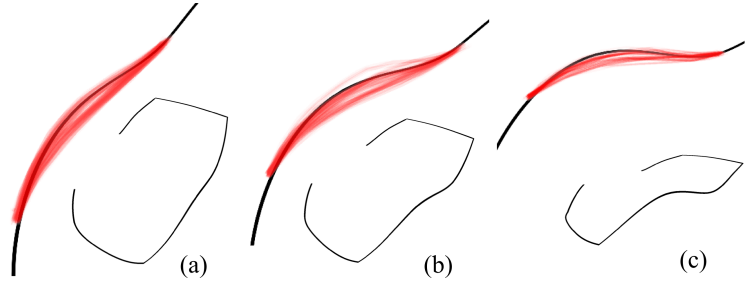

Figure 6: Given several views of a mirror-symmetric surface, subjects drew the missing, symmetric portion of each outline curve from 3 views $(a-c)$. Even with boundary conditions available, a consistent bias towards a "flatter" silhouette is clearly visible.

Finally, we presented the same three views as above, crossed with four different rendering conditions, and asked subjects to draw the curve along the center of the surface lying in the symmetry plane. The rendering conditions were outline, shaded, outline with four left-to-right contours, and outline+contours with additional ticks at the contour midpoints. The motivation here was to explore how drawing accuracy increased as more information was provided, to the point where the curve was almost fully specified. Results of this test are visualized in Figure 7.

We theorize that artists either try to draw the center-line along the surface, or the surface profile in the symmetry plane. Figure 7 shows that projecting onto the surface with greater foreshortening results in lower accuracy. At the endpoints of the curve, there is a clear bias towards the viewpoint which grows as foreshortening increases. On the interior, subjects again tended to 'flatten' their curves.

Interestingly, we found that results in shaded and outline+contour conditions did not deviate significantly from the outline-only condition. One of our concerns with using a freeform surface was that subjects would not understand the actual 3D shape, and so would be imagining the "wrong" surface when presented with a different view and no internal surface information. That the additional context provided by shading and cross-contours did not significantly improve performance increases our confidence that subjects did understand the 3D shape from our example views.

When provided with tick-marks at the center of each cross-contour, accuracy was, as expected, greatly increased. In the most foreshortened case there is still a significant deviation from the correct result, suggesting that subjects were largely connecting-the-dots.

Overall, we observed consistent biases in all conditions, confirming results found in previous human perception studies and indicating that expert $3 \mathrm{D}$ artists are not immune to the perceptual biases repeatedly observed in previous work. However, we did find that 3D error increases with increased foreshortening. This differs from claims made by works which only consider 2D image-space metrics, where it is 
found that error is largest in the $25-55^{\circ}$ range [HKJR08]. Although image-space error may decrease, the smaller errors are magnified when projected onto increasingly foreshortened 3D surfaces. Hence, the error in 3D depiction is larger, even if absolute 2D error magnitude is smaller.

\section{Implications for Design}

Various works in the SBM literature have reported that test subjects have had difficulties or produced unexpected results using 3D curve sketching techniques $\left[\mathrm{CMZ}^{*} 99, \mathrm{GBK}^{*} 02\right.$, KHR04]. It seems likely that these difficulties are in part due to fundamental perceptual limitations. In this section we consider how foreshortening bias may impact existing 3D curve sketching techniques, and suggest some potential avenues for future work.

Observational studies of designers and other artists have noted that curves are often over-sketched many times, possibly to explore minor variations or correct for drawing errors [Bau94, BKK03]. It has been proposed that averaging such over-sketches will improve drawing accuracy [KS07, BBS08]. While correspondence to the artists intended image-space curve may improve, since the distribution of foreshortening error is biased the average curve will not converge on the desired $3 \mathrm{D}$ result.

Bae et al. [BBS08] suggest that "a good view for sketching...is one where the sketch surface has a large visible projection". Figure 7 implies that there is "no free lunch" when drawing projections of $3 \mathrm{D}$ curves, as there is no single viewpoint in which subjects could specify both depth and 3D shape without making significant errors. Essentially, the curve must be drawn on multiple surfaces, and maximizing the visible area of one will obscure the other.

Consider the simplest approach of drawing onto a 3D plane. Clearly one should sketch from a viewing direction nearly orthogonal to the plane. However, the plane position and orientation should be specified from a perpendicular viewpoint. Hence, at least one, and perhaps two significant view rotations will be necessary to mitigate foreshortening error when drawing a single 3D curve.

Single-view multi-curve freeform creation techniques [CMZ*99, KS07, BBS08] will suffer most from perceptual bias. Bae et al.'s sketchability metric [BBS08] suggests that $45^{\circ}$ viewpoints are optimal for these types of techniques. However, [HKJR08] notes that image-space bias is largest in these viewing directions, and our results show that the $3 \mathrm{D}$ error is also significant.

The situation is more complex for freeform multi-view creation techniques, in which the two ray-fans passing through the sketched strokes are intersected to define a 3D curve [KHR04, KS07, BBS08]. Drawing from two highly orthogonal views will allow both depth and shape to be specified with little ambiguity, although this may limit the space of drawable curves to those which project without overlap into both views. Since these techniques generally take both curves into account when determining depth and shape, if either curve is drawn from some arbitrary view, the fused result will likely diverge from what the artist intended.

While drawing context can sometimes help fix the endpoints of the sketched curves, this would not resolve any of the ambiguity in our study conditions. Hence, practical systems which attempt to infer freeform curves from 2D strokes will need to support incremental correction from additional viewing directions, as proposed by [KS07]. However, some care must be taken, because "correcting" from views in which significant bias is present may actually serve to increase error. Techniques for constraining the current 3D curve before corrective strokes are integrated would help here, and have yet to be explored in the SBM literature.

Although constraint interfaces are often tedious, artists make extensive use of visual image-space constraints, such as tick-marks, guidelines, and so on. One possibility would be to detect such marks and snap them to constrained positions, such as edge midpoints, equal 3D lengths, and so on. Interactive visual feedback on the relationship between the current stroke and the scene context would also be useful. For example, the cursor could indicate when the pen hovers over edge midpoints, or show perpendicular directions when the pen is near an existing edge. Such transient visual guides may serve much the same function as explicit constraints, resulting in strokes that are more accurate without significantly complexifying the interface. We believe this is an interesting space for future exploration.

In general, it seems clear that 3D curve creation, evaluation and editing will necessarily involve extensive view rotation. This statement is supported by the extremely frequent view rotation seen in the activity plots of [BBS08]. The automatic view rotation in that work is a good starting point, however what constitutes effective view rotation assistance has not been explored. Here again the perception literature can provide some guidance. [BL08] shows that the $10-15^{\circ}$ effective view rotations provided by stereovision and head tracking do not significantly improve accuracy in estimation of depth measurements. Rotations of at least $45^{\circ}$ are necessary to improve perception of the metric structure of 3D shapes, and furthermore that continuous rotation between viewpoints is necessary, rather than discrete views.

An interesting result from our study is that the same perceptual bias was observed when subjects drew on a shaded surface as in the outline-only condition. Drawing directly onto 3D surfaces has historically been used in a wide range of modeling interfaces, including virtually every commercial NURBS modeler. As freeform surfaces often have complex variations in curvature, foreshortening bias will vary locally over the projected image. Hence, although technically simple, avoiding error of intent when drawing onto 3D surfaces is likely to be very difficult even for skilled artists. 


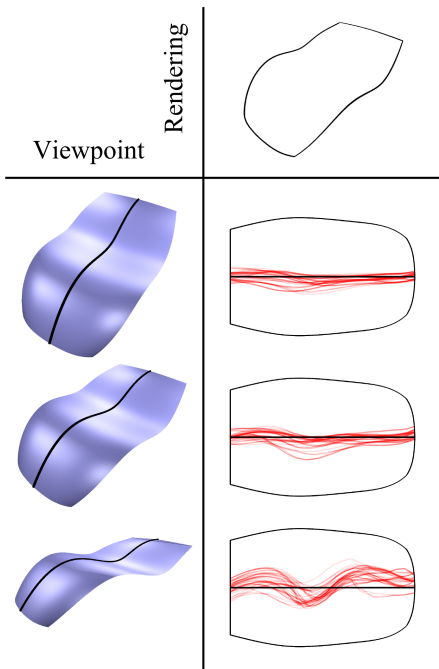

(a)
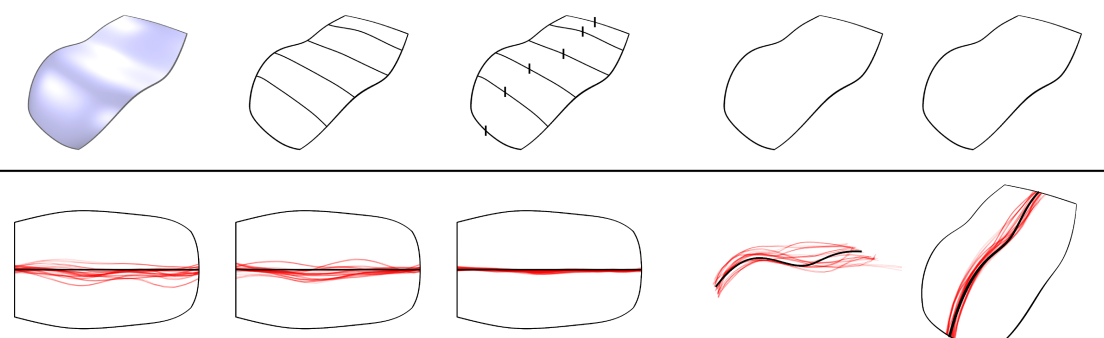

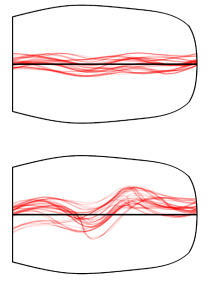

(b)

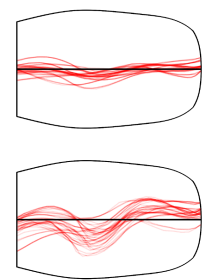

(c)

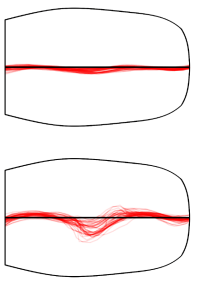

(d)
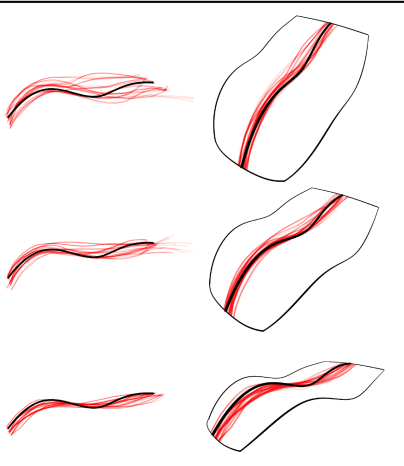

(e)

(f)

Figure 7: Subjects attempted to draw a curve lying on a freeform surface which also lies in the plane of symmetry. Projections of the sketched curves onto the surface are shown for the outline (a), shaded (b), outline+contour (c), and tickmark (d) conditions, crossed with the three viewing angles. Column (e) shows projections onto the symmetry plane in the outline condition, and column $(f)$ shows the image-space results. In the face-on view (upper row) the curves are straighter but the profile is less accurate, while in the edge-on view (bottom row) we observe the opposite. The intermediate view (middle row) exhibits significant error in both projections. Even when tick-marks along the central curve were provided, significant deviations were observed the edge-on view (d, bottom row).

\subsection{Conclusion}

Despite the availability of fully digital design pipelines, workflows based on physical 3D models are still widely used in architecture and design. The perceptual studies we have reviewed suggest that, rather than being due to a lack of technical sophistication, this behavior is highly sensible - computer projections clearly are not to be trusted.

Our study explores the errors expert 3D artists make when depicting foreshortened projections of 3D curves. We have shown that the distribution of error in expert drawings exhibits a bias similar to that which has been repeatedly observed in prior studies of non-expert subjects. Armed with this knowledge, we analyzed current SBM practices, noting how foreshortening bias will impact various 3D curvedrawing techniques and suggesting potential directions for future exploration.

One limitation of our study is that, as we have found no mathematical definition of "accuracy" which can be applied to freehand curves, we have not objectively quantified drawing errors. Furthermore, the manual tracing protocol used to process the data would add a small bias to any numerical results. As these issues may be resolved in future works, we have made our data available on-line at http: //www. dgp.toronto. edu/ rms/data/CurveDrawing.

Surprisingly, several subjects expressed frustration with our drawing tasks and remarked on how difficult it was to try to draw accurate freehand curves. We note that these are exactly the kinds of drawing tasks involved in most SBM systems. This may indicate that tools which require the designer to draw even moderately accurate projections from arbitrary viewpoints, without any external constraints, may not lead to proposed increases in efficiency. Anecdotally, we have found that many skilled artists are quite confident of their drawing abilities, so when errors of intent occur, they may assume that the system is broken. This could be another potential barrier to adoption of SBM systems.

One obvious way to enhance SBM systems would be to account for perceptual bias within future 3D inference techniques. From an interface standpoint this may be problematic, as significant image-space corrections will need to be applied (Consider the cube drawings in Figure 1). The issue is that the resulting drawing may "look wrong" to the designer. Hence, a more effective approach may be to account for perceptual bias in the projection of the existing 3D geometry. While we can provide no clear idea of how to accomplish this, natural scene statistics [NLC*00, YP03, HP05] would appear to provide a reasonable starting point.

\section{Acknowledgements}

The authors would like to thank Seok-Hyung Bae for his assistance with this work. The first and fourth authors are supported in part by NSERC and MITACS. 


\section{References}

[Bau94] BAUDEL T.: A mark-based interaction paradigm for freehand drawing. In Proc. UIST'94 (1994), pp. 185-192.

[BBS08] BAE S.-H., BALAKRISHNAN R., SINGH K.: ILoveSketch: as-natural-as-possible sketching system for creating 3D curve models. In Proc. UIST '08 (2008), pp. 151-160.

[BKK03] BAE S.-H., KIM W.-S., KWON E.-S.: Digital styling for designers: Sketch emulation in computer environment. In Proc. ICCSA (2003), pp. 690-700.

[BL08] Bingham G., Lind M.: Large continuous perspective transformations are necessary and sufficient for accurate perception of metric shape. Perception \& Psychophysics 70, 3 (2008).

[CB97] Cohen D., Bennet S.: Why can't most people draw what they see? J. Exp. Psych.: Human Percep. and Perf. 23, 3 (1997), 609-621.

[CGL*08] Cole F., Golovinskiy A., Limpaecher A., BarRos H., Finkelstein A., FunkHouser T., RuSinkieWICZ S.: Where do people draw lines? ACM Trans. Graph. 27, 3 (2008), 1-11.

[CGL*09] Cole F., Golovinskiy A., Limpaecher A., BARRos H., Finkelstein A., Funkhouser T., RUSinkieWICZ S.: Line drawings of $3 \mathrm{~d}$ shapes, 2009. http://www.cs. princeton.edu/gfx/proj/ld3d/.

[CKX*08] Chen X., Kang S. B., Xu Y.-Q., Dorsey J., SHUM H.-Y.: Sketching reality: Realistic interpretation of architectural designs. ACM Trans. Graph. 27, 2 (2008), 1-15.

[CMZ*99] COHEN J. M., Markosian L., ZelezniK R., Hughes J. F., BARZEL R.: An interface for sketching 3D curves. In Proc. I3D '99 (1999), pp. 17-21.

[DXS*07] Dorsey J., Xu S., Smedresman G., RushmeIER H., MCMillan L.: The mental canvas: A tool for conceptual architectural design and analysis. In Proc. Pacific Graphics (2007).

[GBK* 02] Grossman T., BalaKrishnan R., KURTENBACH G., Fitzmaurice G., Khan A., BuXton B.: Creating principal 3d curves with digital tape drawing. In Proc. CHI 'O2 (2002), pp. 121-128.

[Goo09] GooGLE INC.: SketchUp 7, 2009. http://sketchup. google.com.

[GRMS01] Gooch B., Reinhard E., Moulding C. ShIRLEY P.: Artistic composition for image creation. In Proc. Eurograph. Work. on Render. Techn. (2001), pp. 83-88.

[HKJR08] Hammad S., Kennedy J., JuRiceVic I., Rajani S.: Ellipses on the surface of a picture. Perception 37, 504-510 (2008).

[HP05] Howe C., PuRves D.: Natural-scene geometry predicts the perception of angles and line orientation. Proc. National Academy of Sciences 102, 4 (2005), 1228-1233.

[IMT99] Igarashi T., Matsuoka S., TAnaka H.: Teddy: a sketching interface for 3D freeform design. In Proc. SIGGRAPH '99 (1999), pp. 409-416.

[Kal05] KALliO K.: 3d6b editor: Projective 3d sketching with line-based rendering. In Proc. SBIM '05 (2005).

[KH06] Karpenko O., Hughes J.: SmoothSketch: 3D freeform shapes from complex sketches. ACM Trans. Graph. 25, 3 (2006), 589-598.

[KHR04] KaRPENKO O., Hughes J., RASKAR R.: Epipolar methods for multi-view sketching. In Proc. SBIM '04 (2004).

[Koz01] Kozbelt A.: Artists as experts in visual cognition. Visual Cognition 8, 6 (2001), 705-723.
[KS07] KARA L. B., SHIMADA K.: Sketch-based 3D-shape creation for industrial styling design. IEEE Comput. Graph. Appl. 27, 1 (2007), 60-71.

[LFG08] LEE S., FENG D., GoOCH B.: Automatic construction of 3D models from architectural line drawings. In Proc. I3D '08 (2008), pp. 123-130.

[MA08] MatThews W., AdAMs A.: Another reason why adults find it hard to draw accurately. Perception 37 (2008), 628-630.

[ML05] MASRY M., LIPSON H.: A sketch-based interface for iterative design and analysis of $3 \mathrm{D}$ objects. In Proc. SBIM '05 (2005), pp. 109-118.

[MRAR05] Mitchell P., RopaR D., ACKROYD K., RAJENDRAN G.: How perception impacts on drawings. J. Exp. Psych.: Human Percep. and Perf. 31, 5 (2005), 996-1003.

[NISA07] NEALEN A., Igarashi T., SORKINE O., AleXA M. Fibermesh: designing freeform surfaces with $3 \mathrm{~d}$ curves. ACM Trans. Graph. 26, 3 (2007).

[NK93] NiCHOlls A., KennedY J.: Foreshortening and the perception of parallel projections. Percep. \& Psychophys. 54, 5 (1993), 665-674.

[NK95] NiCHOlls A., Kennedy J.: Foreshortening in cube drawings by children and adults. Perception 24 (1995), 14431456 .

[NLC*00] Nundy S., Lotto B., Coppola D., Shimpi A., Purves D.: Why are angles misperceived? Proc. National Academy of Sciences 97, 10 (2000), 5592-5597.

[OSD06] OH J.-Y., Stuerzlinger W., DANAhy J.: Sesame: towards better 3D conceptual design systems. In Proc. DIS '06 (2006), pp. 80-89.

[Pug92] Pugh D.: Designing Solid Objects Using Interactive Sketch Interpretation. In Proc. I3D '92 (1992), pp. 117-126.

[RL95] REITH E., LIU C. H.: What hinders accurate depiction of projective shape? Perception 24 (1995), 995-1010.

[SWSJ05] Schmidt R., Wyvill B., Sousa M., Jorge J.: Shapeshop: Sketch-based solid modeling with blobtrees. In Proc. SBIM '05 (2005), pp. 53-62.

[TBSR04] TSANG S., BALAKRISHNAN R., Singh K., RANJAN A.: A suggestive interface for image guided $3 d$ sketching. In Proc. CHI 'O4 (2004), pp. 591-598.

[TM97] TAYLOR L., MitChELL P.: Judgements of apparent shape contaminated by knowledge of reality: Viewing circles obliquely. British J. Psych. 88 (1997), 653-670.

[Tod08] ToDoROVIC D.: Is pictoral perception robust? the effect of the observer vantage point on the perceived depth structure of linear-perspective images. Perception 37 (2008), 106-125.

[Too99] ToOMELA A.: Drawing development: Stages in the representation of a cube and a cylinder. Child Development 70, 5 (1999), 1141-1150.

[VTMS04] Varley P., Takahashi Y., Mitani J., Suzuki H.: A two-stage approach for interpreting line drawings of curved objects. In Proc. SBIM 'O4 (2004), pp. 117-126.

[YP03] YANG Z., PURVES D.: A statistical explanation of visual space. Nature Neuroscience 6, 6 (2003), 632-640.

[ZHH96] ZelezNiK R. C., HeRndon K. P., Hughes J. F. Sketch: an interface for sketching 3D scenes. In Proc. SIGGRAPH '96 (1996), pp. 163-170. 